\title{
Rigid and Flexible Quantification in Plural Predicate Logic*
}

\author{
Lucas Champollion \\ New York University
}

\author{
Justin Bledin \\ Johns Hopkins University
}

\author{
Haoze Li \\ New York University
}

\begin{abstract}
Noun phrases with overt determiners, such as some apples or a quantity of milk, differ from bare noun phrases like apples or milk in their contribution to aspectual composition. While this has been attributed to syntactic or algebraic properties of these noun phrases, such accounts have explanatory shortcomings. We suggest instead that the relevant property that distinguishes between the two classes of noun phrases derives from two modes of existential quantification, one of which holds the values of a variable fixed throughout a quantificational context while the other allows them to vary. Inspired by Dynamic Plural Logic and Dependence Logic, we propose Plural Predicate Logic as an extension of Predicate Logic to formalize this difference. We suggest that temporal for-adverbials are sensitive to aspect because of the way they manipulate quantificational contexts, and that analogous manipulations occur with spatial for-adverbials, habituals, and the quantifier all.
\end{abstract}

Keywords: all, aspectual composition, dependence logic, dynamic plural logic, foradverbials, Plural Predicate Logic, quantification, quantization puzzle, team logic, telicity

\section{Introduction}

It has been known since Garey (1957) that the aspectual properties of a verb phrase should be derived both from the nature of the verb and its arguments. Thus, when a verb like eat combines with different objects, the result can be an atelic verb phrase that is compatible with for-adverbials, as in (1a), or a telic verb phrase that is not, as in (1b). This process is known as aspectual composition.

(1) a. John ate $\{$ apples / applesauce $\}$ for an hour.

b. *John ate $\{$ two apples / two cups of applesauce $\}$ for an hour.

Early theories of aspectual composition, like Verkuyl (1972), captured this process via syntactic features that are passed on from the verb and the object to the verb

* We thank Fredrik Engström and Anna Szabolcsi for helpful discussion and comments. 
phrase. Dowty (1979) criticized such feature-based approaches as lacking explanatory power. In model-theoretic approaches, like Krifka $(1989,1998)$, telicity is rather correlated with algebraic/mereological notions, and it is the semantic properties of the verb and of the object that determine those of the verb phrase. In (1b), the object noun phrase is quantized: no proper part of a sum of two apples, for example, is a sum of two apples. Moreover, eat is an incremental-theme verb: the themes of the subevents of any eating event $e$ are proper parts of the theme of $e$. As a result, the full verb phrase is also quantized - no subevent of any event of eating two apples is itself an event of eating two apples - and so this verb phrase is incompatible with for an hour. In contrast, the bare object noun phrases in (1a) are not quantized. For example, a proper part of a sum of apples may well be a sum of apples; hence eat apples is not quantized either-and so it is compatible with for an hour.

On this type of approach, a verb phrase built from an incremental-theme verb and an object inherits the quantization properties of its object. The judgments in (1) can then be explained by equating telicity with quantization and restricting the domain of application of for-adverbials to non-quantized predicates, or to predicates that meet some stronger condition, such as the subinterval property in Dowty (1979) or stratified reference in Champollion (2017). However, many noun phrases that are prima facie non-quantized can combine with incremental-theme verbs to form telic predicates (Mittwoch 1982; Egg 1994; White 1994; Eberle 1998; Naumann 1999; Zucchi \& White 2001); this has come to be known as the "quantization puzzle", (Filip 2000).
a. *John ate some apples for an hour.
b. *John ate less than three apples for an hour.
c. *John ate a quantity of apples for an hour.

These facts suggest that telicity has less to do with quantization per se than with a more general distinction between noun phrases with overt determiners, like those in (1b) and (2), and bare noun phrases, like those in (1a).

These two kinds of noun phrases also pattern differently in other kinds of environments. First, there are similar co-occurrence patterns with spatial measure adverbials and indefinite quantity adverbials:

(3) $\{$ Trees $/ *$ Some trees $\}$ grow for miles around this castle. (Moltmann 1991)

(4) He read $\{$ poetry $/ *$ something $\}$ a lot.

(Mittwoch 1982)

Second, in sentences like (5), a habitual interpretation is preferred only with the bare nominal that can pick out multiple articles:

(5) John wrote $\{$ copy / an article $\}$ for the Times.

(Mittwoch 1982) 
Rigid and Flexible Quantification in Plural Predicate Logic

Another related contrast crops up with all:

a. All the guests are Canadians.

b. *All the guests are $\{$ some / five / several $\}$ Canadians.

Taken together, these observations suggest a difference between bare plurals and bare mass nouns on the one hand, and other indefinite and cardinal noun phrases on the other hand. The fact that a varied set of constructions distinguishes between these two kinds of noun phrases and draws the line between them in the same place motivates positing a semantic difference between the two kinds. The puzzle is how to formally capture the contrast between the two kinds of noun phrases in a way that explains how they affect the relevant properties of the verb phrase in different ways.

We will refer to bare plurals and mass terms as unspecified noun phrases and to indefinite and cardinal noun phrases with overt determiners as specified noun phrases. Pretheoretically, it is not clear what the semantic difference is between these two kinds of noun phrases that can explain the above patterns. Krifka (1998: §3.8) suggests, following Mittwoch (1982), that the difference between them is scope-based: specified noun phrases must take wide scope over the verb phrase and its modifiers, whereas unspecified noun phrases can take narrow scope. However, Zucchi \& White (2001: §5) argue convincingly against this kind of account because when a distributive quantifier intervenes between a for-adverbial and a specified noun phrase, that noun phrase can be interpreted in a way that suggests a narrow-scope position. Thus, in (7a), one golf ball must have been hit and retrieved repeatedly, but in (7b) it is also possible that each time a different ball was hit (Zucchi p.c. to van Geenhoven 2005):

a. Jim hit a golf ball into the lake for an hour.

b. Jim hit a golf ball into the lake every five minutes for an hour.

This suggests that the specified noun phrase a golf ball can take scope under the distributive quantifier every five minutes as well as under the for-adverbial.

In fact, as shown in Champollion (2016a) and the literature cited there, covariation is even possible under certain circumstances when distributivity is contributed by a contextually licensed covert operator. In a context where the daily intake of pills by hospital patients is salient, the following example can be interpreted as involving covariation of two pills with the for-adverbial:

(8) The patient took two pills for a month and then went back to one pill.

These kinds of facts make it difficult to maintain that the different behavior of the relevant noun phrases is driven by scope. In this paper, we suggest instead that specified and unspecified noun phrases are distinguished by what we call their rigid 
versus flexible quantificational profiles. ${ }^{1}$ The intuitive idea is the following. When a variable is bound by a specified noun phrase, the values assigned to it are held fixed, or rigidified, throughout a quantificational context; in (2), this causes a certain quantity of apples to be held fixed throughout the subintervals introduced by for an hour. In contrast, unspecified noun phrases involve flexible existential quantification where the values assigned to a variable can fluctuate throughout a context. Building on the verb semantics in Krifka (1998), we then offer a more refined explanation of how the verb interacts with the object noun phrase to derive the aspectual properties of the verb phrase. By formalizing the semantic difference between specified and unspecified noun phrases in this way, we are able to avoid the quantization puzzle because we do not tie the behavior of these noun phrases only to algebraic properties such as quantization or stratified reference.

In Section 2, we formalize the rigid-flexible dichotomy by enriching Predicate Logic with a notion of quantificational context that replaces assignment functions by sets of assignment functions. In Section 3, we use this system to explain the judgments in (1) and (2). Section 4 explains how distributivity operators such as every five minutes can mask the rigidity requirements imposed by specified noun phrases and how this allows for covariation to arise. In Section 5, we show how the rigid-flexible dichotomy can also explain the contrasts in the related examples (3) through (6); among other things, we suggest that all manipulates quantificational contexts along the same lines as for-adverbials. After comparing our approach in Section 6 to earlier work on the quantization puzzle by Zucchi \& White (2001), we conclude in Section 7.

\section{Rigidity in Plural Predicate Logic}

Inspired by Dynamic Plural Logic (DPIL, van den Berg 1996; Brasoveanu 2008, 2013; Henderson 2014) and by Dependence Logic and Team Logic (Väänänen 2007), we propose Plural Predicate Logic (PPL) to formalize rigid and flexible existential quantification. The essential difference between PPL and ordinary predicate logic (PL) is that whereas formulae in PL are evaluated relative to single assignments, PPL uses sets of assignments, which we refer to as quantificational contexts.

Because our examples involve distribution over time, we will work with firstorder models of the form $\mathfrak{M}=\langle\mathfrak{D}, \mathfrak{I}\rangle$ where the domain $\mathfrak{D}=\mathfrak{D}_{e} \cup \mathfrak{D}_{\tau}$ consists of a subdomain $\mathfrak{D}_{e}$ of individuals and a subdomain $\mathfrak{D}_{\tau}$ of time intervals, and $\mathfrak{I}$

1 The correspondence between rigidity and the presence of overt determiners is not one to one. For one thing, languages such as French use determiners to express the meaning of English bare plurals and bare mass terms. For another, the negative polarity item any cannot be treated as introducing rigid quantification; otherwise, we would incorrectly predict that sentences like John didn't eat any apples for an hour are infelicitous. Thanks to Anna Szabolcsi for this observation. 
Rigid and Flexible Quantification in Plural Predicate Logic

is a standard interpretation function where $\mathfrak{I}(R) \subseteq \mathfrak{D}^{n}$ for any $n$-ary relation $R$. We assume that $\mathfrak{D}_{e}$ and $\mathfrak{D}_{\tau}$ are each subject to the axioms of classical extensional mereology; that is, they are equipped with partial orders $\leq_{e}$ and $\leq_{\tau}$ and sum operations $\oplus_{e}$ and $\oplus_{\tau}$ such that each $\oplus_{i}$ is the least upper bound of its $\leq_{i}$ (for additional details, see Champollion \& Krifka 2016). We assume that temporal intervals may be discontinuous, and that $\leq_{\tau}$ represents temporal inclusion. We do not model other relations such as temporal precedence, but they could easily be added to the system.

Variable assignments in PL are total functions $g_{1}, g_{2}, \ldots$ from the set of variables $V$ to $\mathfrak{D}$. We work with quantificational contexts $G, H, \ldots$, each of which is a nonempty set of variable assignments. Consider for example the following set $G$, consisting of three assignment functions $g_{1}, g_{2}$ and $g_{3}$, where $g_{1}$ assigns Ann to $x$ and Bill to $y, g_{2}$ assigns Carlos to $x$ and Ann to $y$, and $g_{3}$ assigns Ann to $x$ and Carlos to $y$. This assignment is pictured in the following matrix:

\begin{tabular}{c||c|c|c|c}
$G$ & $\ldots$ & $x$ & $y$ & $\ldots$ \\
\hline \hline$g_{1}$ & $\ldots$ & Ann & Bill & $\ldots$ \\
\hline$g_{2}$ & $\ldots$ & Carlos & Ann & $\ldots$ \\
\hline$g_{3}$ & $\ldots$ & Ann & Carlos & $\ldots$
\end{tabular}

In PL, variable assignments are manipulated as shown in (10):

$$
g[x] h:=\text { for any variable } v \text {, if } v \neq x \text { then } g(v)=h(v)
$$

(Assignments $g$ and $h$ differ at most with respect to the value assigned to $x$.)

In contrast, the manipulation of assignments in PPL is based on a relation between sets of assignments, which we define as follows:

$$
G[x] H:=\forall g \in G \exists h \in H \text { such that } g[x] h \text { and } \forall h \in H \exists g \in G \text { such that } g[x] h
$$

For example, let $G$ be as in (9) and let $H$ be as in (12); then $G[z] H$ holds:

\begin{tabular}{c||c|c|c|c|c}
$H$ & $\ldots$ & $x$ & $y$ & $z$ & $\ldots$ \\
\hline \hline$h_{1}$ & $\ldots$ & Ann & Bill & David & $\ldots$ \\
\hline$h_{2}$ & $\ldots$ & Carlos & Ann & Eva & $\ldots$ \\
\hline$h_{3}$ & $\ldots$ & Ann & Carlos & Flora & $\ldots$ \\
\hline$h_{4}$ & $\ldots$ & Ann & Carlos & George & $\ldots$
\end{tabular}

In addition to (11), we define a rigidified notion of assignment manipulation in (13). This notion is the backbone of our analysis. It requires every assignment in the output context $H$ to assign the same value to $x$.

$$
G[x !] H:=G[x] H \text { and } h(x)=h^{\prime}(x) \text { for any } h, h^{\prime} \in H
$$


For example, let $G$ and $H$ be as before; then $G[z !] H$ does not hold because the different assignment functions in (12) disagree on $z$. However, $G[z$ ! $] H^{\prime}$ holds for the following context $H^{\prime}$ :

\begin{tabular}{c||c|c|c|c|c}
$H^{\prime}$ & $\ldots$ & $x$ & $y$ & $z$ & $\ldots$ \\
\hline \hline$h_{1}$ & $\ldots$ & Ann & Bill & David & $\ldots$ \\
\hline$h_{2}$ & $\ldots$ & Carlos & Ann & David & $\ldots$ \\
\hline$h_{3}$ & $\ldots$ & Ann & Carlos & David & $\ldots$ \\
\hline$h_{4}$ & $\ldots$ & Ann & Carlos & David & $\ldots$
\end{tabular}

With this machinery in place, we can now lift the interpretation function $\mathfrak{I}$ in a model $\mathfrak{M}$ to an interpretation function for all well-formed formulae in the language that evaluates them for truth relative to models and quantificational contexts. (One can then define truth at a model in terms of truth at this model and every quantificational context: $\llbracket \varphi \rrbracket_{M}=T$ iff for all $G, \llbracket \varphi \rrbracket_{M}^{G}=T$.)

We first define the semantics of atomic formulae in (15). Such formulae are interpreted pointwise on each assignment (for ease of exposition, we leave the relativity to a model implicit):

Interpretation of atomic formulae

a. $\llbracket \llbracket R\left(x_{1}, \ldots x_{n}\right) \rrbracket^{G}=T$ iff for all $g \in G,\left\langle g\left(x_{1}\right), \ldots g\left(x_{n}\right)\right\rangle \in \mathfrak{I}(R)$

b. $\llbracket x_{1}=x_{2} \rrbracket^{G}=T$ iff for all $g \in G, g\left(x_{1}\right)=g\left(x_{2}\right)$

Moving on to the sentential connectives, the interpretation of conjunction is straightforward:

Interpretation of conjunction

a. $\quad \llbracket \varphi \wedge \psi \rrbracket^{G}=T$ iff $\llbracket \varphi \rrbracket^{G}=T$ and $\llbracket \psi \rrbracket^{G}=T$

However, other connectives present us with some interesting choices (see Galliani 2017 for an overview). In dependence logic, team logic, and their variants, negation can be defined in the following ways:

Interpretation of negation

$$
\begin{array}{ll}
\text { a. } & \llbracket \neg \varphi \rrbracket^{G}=T \text { iff } \llbracket \varphi \rrbracket^{G}=F \\
\text { b. } & \llbracket \sim \varphi \rrbracket^{G}=T \text { iff } \llbracket \varphi \rrbracket^{G^{\prime}}=F \text { for all } G^{\prime} \subseteq G
\end{array}
$$

In (15a), negation simply flips between truth and falsity at a quantificational context. In (15b), however, the truth value of a negated formula $\sim \varphi$ relative to context $G$ depends on whether the embedded sentence is false at $G$ and all other subcontexts of $G$.

Likewise, we can define disjunction in at least two ways. The second option is 
Rigid and Flexible Quantification in Plural Predicate Logic

sometimes known as splitjunction in dependence logic (see also Hawke \& SteinertThrelkeld 2016).

\section{Interpretation of disjunction}

a. $\quad \llbracket \varphi \vee \psi \rrbracket^{G}=T$ iff $\llbracket \varphi \rrbracket^{G}=T$ or $\llbracket \psi \rrbracket^{G}=T$

b. $\llbracket \varphi \otimes \psi \rrbracket^{G}=T$ iff there exists two (possibly empty) sets $G^{\prime}$ and $G^{\prime \prime}$ such that $G=G^{\prime} \cup G^{\prime \prime}$ and $\llbracket \varphi \rrbracket^{G^{\prime}}=T$ and $\llbracket \psi \rrbracket^{G^{\prime \prime}}=T$

The availability of these alternative negations and disjunctions raises interesting questions about which of these formal connectives are best suited for translating ordinary language expressions involving negation and/or disjunction. But since we focus in this paper on examples which do not involve negation or disjunction, we do not pursue this further here (though we say a bit more about this issue in the conclusion).

We assume that the object language has the means to talk about the cardinality of individuals in $\mathfrak{D}_{e}$. More specifically, an individual in $\mathfrak{D}_{e}$ is an atom just in case its only mereological part is itself: $\operatorname{atom}(x):=\forall y \leq_{e} x[y=x]$. We assume that every individual in $\mathfrak{D}_{e}$ is the sum of a set $S \subseteq \mathfrak{D}_{e}$ of one or more atomic individuals. For any $x \in \mathfrak{D}_{e}$, we write $|x|$ for the number of atoms that $x$ consists of. Based on this, we define domain-level cardinality predicates as follows:

\section{Domain-level cardinality}
a. $\llbracket \llbracket x=n \rrbracket^{G}=T$ iff for all $g \in G,|g(x)|=n$
b. $\quad \llbracket x \leq n \rrbracket^{G}=T$ iff for all $g \in G,|g(x)| \leq n$
c. $\quad \llbracket x \geq n \rrbracket^{G}=T$ iff for all $g \in G,|g(x)| \geq n$

Finally, we reach the crucial clauses for quantification. Building on the flexible and rigid manipulation of assignments in (11) and (13), we define two types of existential quantification: flexible existential quantification, as in (20a), and rigid existential quantification, as in (20b).

a. $\quad \llbracket \exists^{\text {flex }} x[\varphi](\psi) \rrbracket^{G}=T$ iff $\llbracket \varphi \wedge \psi \rrbracket^{H}=T$ for some $H$ such that $G[x] H$

b. $\quad \llbracket \exists^{\text {rigid }} x[\varphi](\psi) \rrbracket^{G}=T$ iff $\llbracket \varphi \wedge \psi \rrbracket^{H}=T$ for some $H$ such that $G[x !] H$

The contrast between these two forms of existential quantification is the key to our solution to the quantization puzzle.

\section{Solving the quantization puzzle: application to for-adverbials}

As we have seen in Section 1, incremental-theme verbs combine with unspecified noun phrases to form atelic verb phrases that are compatible with for-adverbials, and with specified noun phrases to form telic verb phrases that are incompatible: 
a. John ate apples for an hour.

b. *John ate two apples for an hour.

We analyze unspecified noun phrases as flexible existential quantifiers and specified noun phrases as rigid existential quantifiers. Thus, the bare noun phrase apples and the specified noun phrase two apples are translated as in (22). As is standard in the literature on plural semantics (Link 1983), for any predicate (or relation) symbol $P$, we write ${ }^{*} P$ for its (pointwise) closure under mereological sum.

$$
\begin{array}{ll}
\text { a. } & {\left[\text { DP } \emptyset^{x} \text { apples }\right](\varphi) \rightsquigarrow \exists^{\text {flex }} x\left[{ }^{*} \operatorname{apple}(x)\right](\varphi)} \\
\text { b. } & \operatorname{two}^{x}[\operatorname{apples}](\varphi) \rightsquigarrow \exists^{\text {rigid }} x\left[{ }^{*} \operatorname{apple}(x) \wedge|x|=2\right](\varphi)
\end{array}
$$

We translate for an hour as follows:

$$
\text { for an } \operatorname{hour}^{t, t^{\prime}}(\varphi) \rightsquigarrow \exists^{\mathrm{rigid}} t[\operatorname{hour}(t)=1]\left(\exists^{\mathrm{flex}} t^{\prime}\left[t^{\prime}<{ }_{\tau} t \wedge t \stackrel{\oplus}{=} t^{\prime}\right](\varphi)\right)
$$

Here, the embedded indefinite an hour is translated as a rigid existential quantifier that introduces an hour-long temporal interval $t$, keeping it constant across the quantificational context. We assume that the for-adverbial also introduces a set of proper subintervals $t^{\prime}$ of $t$ that jointly cover $t$. Letting $G(x)$ for the set $\{g(x): g \in G\}$, the "cover" relation, which is symmetric, is defined as follows:

$$
\llbracket x \stackrel{\oplus}{=} y \rrbracket^{G}=T \text { iff } \bigoplus G(x)=\bigoplus G(y)
$$

Since the variable $t^{\prime}$ is bound flexibly, each assignment in the context is free to map it to a different value. In effect, (23) unfurls the time interval introduced by the indefinite an hour by spreading different subintervals of this interval across the individual assignments in the quantificational context. The for-adverbial then passes $t^{\prime}$ rather than $t$ to the verb phrase $\varphi$. Since ordinary lexical predicates are evaluated at each assignment in the context, these predicates will be required to hold at each of the intervals over which $t^{\prime}$ ranges. $^{2}$

We assume that (21a) is compositionally translated as follows:

$$
\begin{aligned}
& \text { John ate apples for an hour } \rightsquigarrow \\
& \exists^{\text {rigid }} t[\operatorname{hours}(t)=1]\left(\exists^{\text {flex }} t^{\prime}\left[t^{\prime}<_{\tau} t \wedge t \stackrel{\oplus}{=} t^{\prime}\right]\left(\exists^{\text {flex }} x\left[{ }^{*} \text { apple }(x)\right]\left({ }^{*} \text { eat }\left(j, x, t^{\prime}\right)\right)\right)\right)
\end{aligned}
$$

The effect of this translation is that (21a) is true relative to a quantificational context $G$ just in case one can find a context $H$ which is just like $G$ except that (i) all of the

2 We could implement stronger notions of atelicity by changing the definition in (23). For example, we could let $t^{\prime}$ range over all the subintervals of $t$ if we wanted to represent atelicity via the subinterval property; but this would run into the minimal-parts problem (Dowty 1979). The definition we use is based on Piñón (2015) and Champollion (2015, 2017). 
assignments in $H$ map $t$ to the same hour-long interval, (ii) each assignment in $H$ maps $t^{\prime}$ to some proper subinterval of that interval, such that the various values of $t^{\prime}$ across $H$ sum up to the value of $t$ (since the subintervals are required to be proper, the assignments cannot all map $t^{\prime}$ to the same subinterval), and (iii) each assignment in $H$ maps $x$ to some potentially different apple or sum of apples that John eats at $t^{\prime}$. Such a quantificational context $H$ is exhibited here:

\begin{tabular}{c||c|c|c|c|l}
$H$ & $\ldots$ & $t$ & $t^{\prime}$ & $x$ & $\ldots$ \\
\hline \hline$h_{1}$ & $\ldots$ & 9pm-10pm & 9pm-9:10pm & apple $_{1}$ & $\ldots$ \\
\hline$h_{2}$ & $\ldots$ & 9pm-10pm & $9: 10 \mathrm{pm}-9: 40 \mathrm{pm}$ & apple $_{2} \oplus$ apple $_{3}$ & $\ldots$ \\
\hline$h_{3}$ & $\ldots$ & 9pm-10pm & $9: 40 \mathrm{pm}-10 \mathrm{pm}$ & apple $_{4}$ & $\ldots$
\end{tabular}

We assume that the lexical semantics of eat specifies that nothing can be eaten more than once; this can be ensured through meaning postulates on verbs as laid out in Krifka (1998). Because of this constraint, the values of $x$ must covary with the values of $t^{\prime}$. The flexible existential quantifier introduced by the bare noun phrase apples allows for this. For example, in the scenario shown in (26), John ate apple ${ }_{1}$ from 9pm to 9:10pm, apple ${ }_{2}$ and apple ${ }_{3}$ from 9:10pm to $9: 40 \mathrm{pm}$, and apple from 9:40pm to $10 \mathrm{pm}$.

Things are different in the case of (21b), where two apples introduces a rigid existential quantifier:

$$
\begin{aligned}
& \text { John ate two apples for an hour } \rightsquigarrow \\
& \exists^{\text {rigid }} t[\text { hours }(t)=1]\left(\exists^{\text {flex }} t^{\prime}\left[t^{\prime}<_{\tau} t \wedge t \stackrel{\oplus}{=} t^{\prime}\right]\right. \\
& \left.\left.\left(\exists^{\text {rigid }} x{ }^{*} \text { apple }(x) \wedge|x|=2\right]\left({ }^{*} \text { eat }\left(j, x, t^{\prime}\right)\right)\right)\right)
\end{aligned}
$$

The truth conditions of this formula differ from those of the previous one with respect to the values that may be assigned to $x$. While the quantifier $\exists^{\text {flex }} x$ in (25) allowed these values to vary from one assignment to the other, this time the quantifier $\exists^{\text {rigid }} x$ requires each assignment in the quantificational context to map $x$ to the same two-apple sum, as in this example:

\begin{tabular}{c||c|c|c|c|c}
$H$ & $\ldots$ & $t$ & $t^{\prime}$ & $x$ & $\ldots$ \\
\hline \hline$h_{1}$ & $\ldots$ & 9pm-10pm & 9pm-9:10pm & apple $_{1} \oplus$ apple $_{2}$ & $\ldots$ \\
\hline$h_{2}$ & $\ldots$ & 9pm-10pm & 9:10pm-9:40pm & apple $_{1} \oplus$ apple $_{2}$ & $\ldots$ \\
\hline$h_{3}$ & $\ldots$ & $9 \mathrm{pm}-10 \mathrm{pm}$ & $9: 40 \mathrm{pm}-10 \mathrm{pm}$ & apple $_{1} \oplus$ apple $_{2}$ & $\ldots$
\end{tabular}

As before, the for-adverbial unfurls the hour-long interval 9pm-10pm into $t^{\prime}$. If the value of $x$ is held fixed across assignments, as shown in (28), the lexical semantics of eat is bound to be violated. In order for (21b) to be true, John would, per impossible, have to eat the same two apples repeatedly over the course of one hour. 
While we have relied on the same assumptions concerning verb semantics as the analysis in Krifka (1998), our treatment of noun phrases is novel. What matters for us is not whether the object noun phrase is quantized, but rather whether it contributes rigid quantification, in the precise sense that we have laid out. This allows us to extend our analysis of the specified and quantized noun phrase two apples to other specified noun phrases such as some apples and less than three apples, even though these noun phrases are not quantized (at least not in any straightforward sense). We assume that indefinites like some apples are translated in the same way as two apples in (22b) except that the cardinality check is different:

$$
\text { some }^{x} \text { [apples] }(\varphi) \rightsquigarrow \exists^{\text {rigid }} x\left[{ }^{*} \text { apple }(x) \wedge|x|>1\right](\varphi)
$$

The rigid quantifier will cause the values of $x$ to be held constant across the quantificational context, which will violate the lexical semantics of eat when a for-adverbial appears in the sentence, as in the previous example (27).

As for modified numerals like less/more than three apples, we assume with Zucchi \& White (2001) and Brasoveanu (2013: §2) that they introduce a maximalization operator that provides access to all the apples that John ate. This is because a sentence like John ate less than three apples is only true if the maximum number of apples that John ate is less than three; it is not enough merely for there to be some set of apples of cardinality less than three that John ate (after all, this might be a proper subset of the apples he ate). This complication has nothing to do with the quantization puzzle in itself; we include it only to get the right truth conditions.

We define maximalization based on the mereological proper-part relation $<_{e}$ between individuals:

$\llbracket \sigma x(\varphi)(\psi) \rrbracket^{G}=T$ iff there is some $H$ such that $G[x !] H, \llbracket \varphi \rrbracket^{H}=T$ and $\llbracket \psi \rrbracket^{H}=T$, and there is no $H^{\prime}$ such that $G[x !] H^{\prime}$ and $\llbracket \varphi \rrbracket^{H^{\prime}}=T$ and $h(x)<_{e}$ $h^{\prime}(x)$ for some $h \in H$ and $h^{\prime} \in H^{\prime}$

This operator first picks a maximally large sum among the entities such that $\varphi$, then rigidly binds this sum to $x$, and finally checks whether $\psi$ holds. Using this operator, we translate the modified numeral less than three apples so as to introduce the maximal number of apples that satisfy the nuclear scope of the quantifier and then use a cardinality test to check that their number is less than three:

$$
\text { less than } \text { three }^{x}\left[\text { apples] }(\varphi) \rightsquigarrow \sigma x\left({ }^{*} \text { apple }(x) \wedge \varphi\right)(|x|<3)\right.
$$

Because this maximalization operator involves rigid assignment manipulation, its effect on for-adverbials is the same as that of the rigid quantifier in (22b).

To sum up, because indefinites and modified numerals introduce rigid quantifiers, our system treats them analogously to unmodified numerals in that they do not allow 
Rigid and Flexible Quantification in Plural Predicate Logic

the value of their variable to vary across a quantificational context, such as the ones unfurled by for-adverbials. Therefore they cannot be used as the theme argument of an incremental-theme verb embedded under a for-adverbial.

\section{Adding distributivity}

Our analysis not only offers a solution to the quantization puzzle, it can also naturally account for the fact that specified noun phrases can covary in the scope of foradverbials when a temporal universal quantifier intervenes. We have seen an example of this in (7); here is another one:

$$
\text { John found a flea (on his dog) every day for a year. }
$$

As we have seen in Section 1, this kind of example is a challenge for scope-based accounts such as the one proposed by Krifka (1998: §3.8), where specified noun phrases must take wide scope over for-adverbials. On such accounts, it is hard to explain why every day can prevent a flea from taking wide scope.

As in DPIL (Brasoveanu 2008; Henderson 2014), we assume that noun phrases of the shape every $N$ launch a separate quantificational context for each $N$. The interpretation of such noun phrases consists of two steps: first, introduce all the $N$ s and spread them out across the quantificational context via an assignment maximalization operator $M$, defined as in (33a); next, distribute over the values of $N$ via an assignment distributive operator $D$, defined as in (33b), which breaks up the quantificational context according to the values of $N$ (see also van den Berg 1996: 98). We write $G_{x=a}$ for the set $\{g \in G: g(x)=a\}$.

$$
\begin{array}{ll}
\text { a. } & \llbracket \mathrm{M} x[\varphi](\psi) \rrbracket^{G}=T \text { iff } \llbracket \varphi \wedge \psi \rrbracket^{H}=T \text { for some } H \text { such that } G[x] H \text { and } \\
\text { there is no } H^{\prime} \text { such that } G[x] H^{\prime} \text { where } \llbracket \varphi \rrbracket^{H^{\prime}}=T \text { and } H(x) \subsetneq H^{\prime}(x) \\
\text { b. } \llbracket \mathrm{D}_{x}(\varphi) \rrbracket^{G}=T \text { iff } \llbracket \varphi \rrbracket^{G_{x=a}}=T \text { for each } a \in G(x)
\end{array}
$$

We translate every day as follows:

$$
\operatorname{every}_{t^{\prime}}^{t^{\prime \prime}}[\operatorname{day}](\varphi) \rightsquigarrow \mathrm{M} t^{\prime \prime}\left[\operatorname{day}\left(t^{\prime \prime}\right) \wedge t^{\prime} \cap t^{\prime \prime} \neq \emptyset\right]\left(\mathrm{D}_{t^{\prime \prime}}(\varphi)\right)
$$

The domain restriction for this quantifier is provided by the time interval stored under $t^{\prime}$; in (32), we assume that the value of this variable is supplied by the rigid indefinite a year. Via $M$, the quantifier (34) then stores the set of all days of $t^{\prime}$ under a new variable $t^{\prime \prime}$, and then distributes the nuclear scope $\varphi$ over $t^{\prime \prime}$ via $D$.

We translate sentence (32) as follows:

$$
\begin{aligned}
& \exists^{\text {rigid }} t[\operatorname{years}(t)=1]\left(\exists ^ { \text { flex } } t ^ { \prime } [ t ^ { \prime } < _ { \tau } t \wedge t \stackrel { \oplus } { = } t ^ { \prime } ] \left(\mathrm{M} t^{\prime \prime}\left[\operatorname{day}\left(t^{\prime \prime}\right) \wedge t^{\prime \prime} \cap t^{\prime} \neq \emptyset\right]\right.\right. \\
& \left.\left(\mathrm{D}_{t^{\prime \prime}}\left(\exists^{\text {rigid }} x\left[{ }^{*} \text { flea }(x)\right]\left({ }^{*} \text { find }\left(j, x, t^{\prime \prime}\right)\right)\right)\right)\right)
\end{aligned}
$$


Effectively, (35) splits up a quantificational context into separate contexts, one for each day, as illustrated here:

\begin{tabular}{|c|c|c|c|c|c|c|}
\hline$H$ & $t$ & $t^{\prime}$ & $t^{\prime \prime}$ & \multirow{3}{*}{$\stackrel{D_{t^{\prime \prime}}}{\longrightarrow}$} & \\
\hline 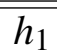 & 2016 & Jan-Mar & day $_{1}$ & & $t^{\prime \prime}$ & $x$ \\
\hline$h_{2}$ & 2016 & Feb-May & day $_{2}$ & & day $_{2}$ & flea $_{2}$ \\
\hline$\ldots$ & $\ldots$ & $\ldots$ & $\ldots$ & & \multirow{2}{*}{\multicolumn{2}{|c|}{$\cdots$}} \\
\hline$h_{1}$ & 2016 & Oct-Dec & day $_{365}$ & & & \\
\hline & & & & & $t^{\prime \prime}$ & $x$ \\
\hline & & & & & $\operatorname{day}_{36}$ & flea, \\
\hline
\end{tabular}

In this example, the indefinite a year is rigid; therefore every assignment assigns to variable $t$ the same value, namely the year 2016. The for-adverbial then assigns the variable $t^{\prime}$ a set of subintervals of that year that cover it. As in previous examples, the division of $t$ into these subintervals is arbitrary; for example, in (36), some of the values of $t^{\prime}$ happen to overlap, but this is not essential. The quantifier every day takes its domain restriction from $t^{\prime}$. It introduces a new variable $t^{\prime \prime}$, which holds all those days that overlap with the values of $t^{\prime}$, that is, all the days of 2016. The distributive operator $D_{t^{\prime \prime}}$ then splits the quantificational context into many separate contexts, one for each day of 2016. As a consequence of this splitting of contexts, the quantifier $a$ flea is free to map its variable $x$ to a different value in each of them even though it is rigid. Since rigidity is checked only within each context, the values of $x$ (the fleas) are free to covary with the values of $t^{\prime \prime}$ (the days).

\section{Application to constructions other than for-adverbials}

We have seen how the distinction between rigid and flexible quantification helps solve the quantization puzzle. However, we have not yet independently motivated the claim that specified noun phrases are rigid while unspecified noun phrases are flexible. To provide this motivation, in this section we show that this distinction also explains the related observations in Section 1 involving spatial and indefinite quantity adverbials, habitual readings, and all.

The analysis of spatial for-adverbials parallels that of temporal for-adverbials, but the quantification is now over regions of space rather than temporal intervals. Consider this slight variant of the earlier example (3) from Moltmann (1991):

a. Trees grow for ten miles around this castle.

b. *Some trees grow for ten miles around this castle. 
Rigid and Flexible Quantification in Plural Predicate Logic

To explain the contrast between (37a) and (37b), we now add a subdomain $\mathfrak{D}_{\sigma}$ of two-dimensional spatial regions to the domain of our models in addition to the subdomains $\mathfrak{D}_{e}$ and $\mathfrak{D}_{\tau}$ of individuals and temporal intervals (where $\mathfrak{D}_{\sigma}$ is also subject to classical mereology). Like for an hour, we analyze for ten miles around this castle as first introducing a spatial region centered at the castle which radiates outward for ten miles, and then unfurling this region into a cover of subregions that are spread throughout a quantificational context:

$$
\begin{aligned}
& \text { for ten miles around this castle }{ }^{s, s^{\prime}}(\varphi) \rightsquigarrow \\
& \exists^{\text {rigid }} s[\text { ten-miles-around-this-castle }(s)]\left(\exists^{\text {flex }} s^{\prime}\left[s^{\prime}<_{\sigma} s \wedge s \stackrel{\oplus}{=} s^{\prime}\right](\varphi)\right)
\end{aligned}
$$

The unspecified noun phrase trees is a flexible quantifier, so we assume that sentence (37a) is translated as follows:

$$
\begin{aligned}
& \exists^{\text {rigid }} s[\text { ten-miles-around-this-castle }(s)]\left(\exists^{\text {flex }} s^{\prime}\left[s^{\prime}<_{\sigma} s \wedge s \stackrel{\oplus}{=} s^{\prime}\right]\right. \\
& \left.\left(\exists^{\text {flex }} x\left[{ }^{*} \operatorname{tree}(x)\right]\left({ }^{*} \text { grow }\left(x, s^{\prime}\right)\right)\right)\right)
\end{aligned}
$$

This sentence effectively tests whether there is some way of assigning potentially different trees (the values of $x$ ) to proper subregions of a space of ten miles around the castle (the values of $s^{\prime}$ ) such that the trees grow in the respective subregions-the intuitive meaning of the sentence.

On the other hand, the specified noun phrase some trees contributes rigid quantification, so (37b) is translated using $\exists^{\text {rigid }} x$ :

$$
\begin{aligned}
& \exists^{\text {rigid }} s[\text { ten-miles-around-this-castle }(s)]\left(\exists^{\text {flex }} s^{\prime}\left[s^{\prime}<\sigma s \wedge s \stackrel{\oplus}{=} s^{\prime}\right]\right. \\
& \left.\left(\exists^{\text {rigid }} x\left[{ }^{*} \operatorname{tree}(x) \wedge|x|>1\right]\left({ }^{*} \operatorname{grow}\left(x, s^{\prime}\right)\right)\right)\right)
\end{aligned}
$$

Now the same sum of trees is passed on as an argument to grow throughout the quantificational context. We assume that it is part of the lexical semantics of grow that nothing can grow in two places at once, hence we have a violation. As in the temporal case, felicity can be restored by intervening with a distributive operator:

a. *Two sentinels are posted for ten miles around this castle.

b. Two sentinels are posted every 100 feet for ten miles around this castle.

By launching separate quantificational contexts for different spatial subregions, every 100 feet facilitates covariation.

It is perhaps unsurprising that spatial and temporal for-adverbials behave so similarly. But, as we have suggested, the difference between rigid and flexible quantifiers is also operative in examples like the following (Mittwoch 1982):

He read \{ poetry / *something \} a lot. 
John wrote $\{$ copy / an article $\}$ for the Times.

We can account for these facts in an analogous fashion as before. Briefly, in (42), a lot sets up a quantificational context where he read \{ poetry / something \} must hold at sufficiently many temporal intervals that are assigned to some variable in the context. The flexible noun phrase poetry can introduce multiple poems while the rigid noun phrase something must introduce a single individual that is held constant throughout the context. This explains the contrast in judgments. Likewise, in (43), the unspecified noun phrase copy can introduce multiple articles throughout the context, and so allows for a habitual reading. But the specified noun phrase an article can introduce only a single article. Assuming that the silent habitual introduces its quantificational context, this would require that John wrote the exact same article over and over again for the Times. This contradicts world knowledge, hence the habitual reading is unavailable.

We leave it for future work to develop these proposals in more depth. Instead, let us finally turn to all, which quantifies over individuals rather than temporal or spatial intervals. We suggest that appealing to rigid and flexible quantification can explain the following variant of the quantization puzzle:

a. All the guests are Canadians.

b. *All the guests are several Canadians.

Our proposal is that all acts like a for-adverbial in introducing the plural referent picked out by its restrictor and then unfurling the quantificational context such that different subindividuals of this referent are assigned throughout the context and passed on to the predicate in the nuclear scope. The literature on the semantics of all is extensive and has covered many different aspects of its behavior, most of which are unrelated to the difference between specified and unspecified noun phrases (e.g. Dowty 1987; Winter 2001; Champollion 2017) . To avoid unnecessary complexity, we do not show how to integrate our approach with existing proposals.

We assume the following entry for all:

$$
\operatorname{all}^{x, x^{\prime}}[\varphi](\psi) \rightsquigarrow \sigma x(\varphi)\left(\exists^{\text {flex }} x^{\prime}\left[x^{\prime}<_{e} x \wedge x^{\prime} \stackrel{\oplus}{=} x\right](\psi)\right)
$$

This has the effect of instantiating $x$ with the maximal sum of all the entities satisfying its restrictor $\varphi$, then flexibly introducing a decomposition of this sum entity, and finally evaluating its nuclear scope $\psi$. The parts of $x$ are stored under $x^{\prime}$ and have to be proper parts of $x$ that sum up to the value of $x$; this forces the values of $x^{\prime}$ to vary throughout the quantificational context that all introduces.

We can then translate (44a) and (44b) as follows: 
Rigid and Flexible Quantification in Plural Predicate Logic

$$
\begin{array}{ll}
\text { a. } & \sigma x\left({ }^{*} \operatorname{guest}(x)\right)\left(\exists^{\mathrm{flex}} x^{\prime}\left[x^{\prime}<_{e} x \wedge x \stackrel{\oplus}{=} x^{\prime}\right]\left(\exists^{\text {flex }} y\left[{ }^{*} \operatorname{Canadian}(y)\right]\left(x^{\prime}=y\right)\right)\right) \\
\text { b. } & \sigma x\left({ }^{*} \operatorname{guest}(x)\right)\left(\exists^{\text {flex }} x^{\prime}\left[x^{\prime}<_{e} x \wedge x \stackrel{\oplus}{=} x^{\prime}\right]\right. \\
& \left.\left(\exists^{\text {rigid }} y\left[{ }^{*} \text { Canadian }(y) \wedge|y|>1\right]\left(x^{\prime}=y\right)\right)\right)
\end{array}
$$

Aside from the cardinality check, these two formulae differ only in that the existential quantifier over $y$ is flexible in (46a) but rigid in (46b). This means that in the first case, the values of $y$ can vary across the quantificational context, while in the second case the value of $y$ is fixed throughout the context. We have translated the copula are as requiring equality between $x^{\prime}$ and $y$; since $x^{\prime}$ has different values throughout the context, $y$ must be able to covary with $x^{\prime}$. This is possible in (46a) but not in (46b), which explains the contrast between (44a) and (44b).

To be sure, there are many puzzles concerning the word all that we have not accounted for in this paper. In the conclusion, we suggest avenues for future research on one of these puzzles.

\section{Previous work: Zucchi \& White (2001)}

The central claim of our approach is that rigidity is a crucial factor that allows noun phrases to lead to telicity even in the absence of quantization. We have assumed essentially the same verb phrase semantics as Krifka (1998) (modulo the event semantics). Zucchi \& White (2001), by contrast, aim to hold on to the idea that quantization is at stake in sentences like these:

a. ??John drank a quantity of milk for an hour.

b. ??John found some fleas on his dog for an hour.

c. ??John found a twig for ten minutes.

a. ??John wrote most of the letters for an hour.

b. ??John found most of the fleas for an hour.

c. ??John found less than half of the fleas for an hour.

To overcome the quantization puzzle, Zucchi \& White explore two strategies, of which the first accounts only for ordinary indefinites as in (47), and the second accounts for both (47) and (48). They leave it open whether it is preferable to use a mix of both strategies or only the second. Their first strategy treats indefinites as predicates over free variables, as in Kamp (1981) and Heim (1982). These variables can be existentially bound by a sentence-level closure operator, but not by foradverbials. Since existential closure takes scope above the for-adverbial, this is similar to a scope account. But Zucchi \& White also assume that the individual variables of indefinites can be existentially bound by distributive quantifiers like every day. This allows them to capture the covariation of indefinites with such quantifiers 
in examples like (7b) and (32). Their second strategy is based on retrieving the maximal number of events of a certain kind within the reference time, following a technique developed by Krifka (1992). For example, an event that can be described as write most of the letters is a writing event whose theme is the sum of all the letters written by John at the reference time $t_{r}$, such that the cardinality of this sum is greater than one half the number of the letters. This reference time variable $t_{r}$ can be supplied at sentence level, or it can be bound by distributive quantifiers such as every day.

Our own approach is similar to Zucchi \& White (2001) in that we use maximization in the interpretation of modified noun phrases. However, we do not assume that every day can directly bind variables supplied by noun phrases. On our approach, the individual variable of a noun phrase is bound by the rigid or flexible quantifier that the noun phrase introduces. The assumption that every day binds the individual variables of noun phrases in its scope strikes us as stipulative. As for the idea that reference time variables are bound by every day, one problem is that as soon as we try to analyze spatial counterparts of the phenomenon, such as the contrast in (41), we need to add an additional spatial counterpart of that variable. No such complication arises in our system because distributive quantifiers like every day and every mile induce covariation via the quantificational contexts that they unfurl rather than via variable binding.

\section{Conclusion and outlook}

We have proposed that noun phrases with overt determiners are distinguished from bare noun phrases by what we have called their rigid versus flexible quantificational profiles. Rigid noun phrases bind a variable and then hold its value fixed throughout a quantificational context, while flexible noun phrases allow these values to vary across the context. To formalize this distinction, we generalized Predicate Logic by relativizing the interpretation function to quantificational contexts, understood as sets of assignment functions. Our proposal was that related phenomena involving temporal and spatial for-adverbials, habituals, and all, among other expressions, can be explained in terms of the way in which these expressions rigidly impact such quantificational contexts.

The formal logic we have defined, Plural Predicate Logic, is a close relative of Dependence Logic and Team Logic (Väänänen 2007) and of Independent Plural Logic (van den Berg 1996). The precise relationship between these logics depends on how we define negation and disjunction, a question we have left open here. At first sight, the version of negation in (17b) seems more adequate than the version in (17a), given that a sentence like For an hour, John didn't dance is true only if there is an hour such that John didn't dance at any of its subintervals. In the case of 
Rigid and Flexible Quantification in Plural Predicate Logic

disjunction, judgments are less clear: the choice between (18a) and (18b) depends on whether sentence (49) has a reading that is compatible with John carrying out both activities in alternation over the course of one hour.

For an hour, John sang or danced.

The entry in (18b) predicts that this reading should be available out of the blue, while the entry in (18a) predicts that sentence (49) can only mean Either John sang for an hour or he danced for an hour, at least in the absence of temporal distributivity operators (Champollion 2016a).

As we have seen, the distinction between rigid and flexible existential quantification allows us to deal with "quantization puzzle"-like phenomena beyond for-adverbials, such as the contrast involving all in (44). There are many puzzles left with "all" that we have not accounted for in this paper. For example, the contrast between Canadians and several Canadians in (44) is reminiscent of the following minimal pair discussed by Zweig (2009):

a. All the linguistics majors dated chemistry majors.

b. All the linguistics majors dated several chemistry majors.

Zweig observes that unlike (50a), (50b) entails that each linguistics major dated several chemistry majors. A full account of the semantics of these sentences requires a more sophisticated treatment of all than the one we have given in (45) and is beyond the scope of this paper; for discussion, see Dowty (1987: 10) and Champollion (2016b, 2017). The distinction between rigid and flexible quantifiers may well play a role in the explanation of this type of difference.

\section{References}

van den Berg, Martin H. 1996. Some aspects of the internal structure of discourse: University of Amsterdam dissertation.

Brasoveanu, Adrian. 2008. Donkey pluralities: Plural information states versus non-atomic individuals. Linguistics and Philosophy 31(2). 129-209. doi:10.1007/s10988-008-9035-0.

Brasoveanu, Adrian. 2013. Modified numerals as post-suppositions. Journal of Semantics 30(2). 155-209. doi:10.1093/jos/ffs003.

Champollion, Lucas. 2015. Refining stratified reference. Theoretical Linguistics 41(3-4). 223-240. doi:10.1515/tl-2015-0015.

Champollion, Lucas. 2016a. Covert distributivity in algebraic event semantics. Semantics and Pragmatics 9(15). 1-65. doi:10.3765/sp.9.15.

Champollion, Lucas. 2016b. Linking the collective-distributive opposition and the 
telic-atelic opposition. In Christopher Hammerly \& Brandon Prickett (eds.), 46th Annual Meeting of the North East Linguistic Society (NELS 46), vol. 1, 171-184. Amherst, MA: University of Massachusetts GLSA Publications. http: //ling.auf.net/lingbuzz/002985/current.pdf.

Champollion, Lucas. 2017. Parts of a whole: Distributivity as a bridge between aspect and measurement, vol. 66 Oxford studies in theoretical linguistics. Oxford, UK: Oxford University Press.

Champollion, Lucas \& Manfred Krifka. 2016. Mereology. In Maria Aloni \& Paul Dekker (eds.), Cambridge handbook of formal semantics Cambridge Handbooks in Language and Linguistics, chap. 13, 369-388. Cambridge, UK: Cambridge University Press. doi:10.1017/cbo9781139236157.014.

Dowty, David R. 1979. Word meaning and Montague grammar: The semantics of verbs and times in generative semantics and in Montague's PTQ. Dordrecht, Netherlands: Reidel. doi:10.1007/978-94-009-9473-7.

Dowty, David R. 1987. Collective predicates, distributive predicates, and all. In Ann Miller \& Zheng-sheng Zhang (eds.), 3rd Eastern States Conference on Linguistics (ESCOL), 97-115. Columbus, OH: Ohio State University.

Eberle, Kurt. 1998. The influence of Plural NPs on the Aktionsart in DRT. In Fritz Hamm \& Erhard Hinrichs (eds.), Plural quantification, Dordrecht, Netherlands: Kluwer. doi:10.1007/978-94-017-2706-8_3.

Egg, Markus. 1994. Aktionsart und Kompositionalität. Berlin, Germany: Akademie Verlag.

Filip, Hana. 2000. The quantization puzzle. In James Pustejovsky \& Carol Tenny (eds.), Events as grammatical objects, from the combined perspectives of lexical semantics, logical semantics and syntax, 3-60. Stanford, CA: CSLI Publications.

Galliani, Pietro. 2017. Dependence logic. In Edward N. Zalta (ed.), The Stanford Encyclopedia of Philosophy, Metaphysics Research Lab, Stanford University spring 2017 edn. https://plato.stanford.edu/archives/spr2017/entries/logic-dependence/.

Garey, Howard B. 1957. Verbal aspect in French. Language 33(2). 91-110. doi:10.2307/410722.

van Geenhoven, Veerle. 2005. Atelicity, pluractionality and adverbial quantification. In Henk J. Verkuyl, Henriëtte de Swart \& Angeliek van Hout (eds.), Perspectives on aspect, vol. 32 Studies in theoretical psycholinguistics, 107-124. Berlin, Germany: Springer. doi:10.1007/1-4020-3232-3_6.

Hawke, Peter \& Shane Steinert-Threlkeld. 2016. Informational dynamics of epistemic possibility modals. Synthese doi:10.1007/s11229-016-1216-8.

Heim, Irene. 1982. The semantics of definite and indefinite noun phrases. Amherst, MA: University of Massachusetts dissertation. http://semanticsarchive.net/ Archive/jA2YTJmN.

Henderson, Robert M. 2014. Dependent indefinites and their post-suppositions. 
Rigid and Flexible Quantification in Plural Predicate Logic

Semantics and Pragmatics 7(6). 1-58. doi:10.3765/sp.7.6.

Kamp, Hans. 1981. A theory of truth and semantic representation. In Jeroen Groenendijk, Theo Janssen \& Martin Stokhof (eds.), Formal methods in the study of language, vol. 135 Mathematical Center Tracts, 277-322. Amsterdam, Netherlands: Mathematisch Centrum. doi:10.1002/9780470758335.ch8.

Krifka, Manfred. 1989. Nominal reference, temporal constitution and quantification in event semantics. In Renate Bartsch, Johan van Benthem \& Peter van Emde Boas (eds.), Semantics and contextual expression, 75-115. Dordrecht, Netherlands: Foris. http://amor.cms.hu-berlin.de/ h2816i3x/Publications/ Krifka\%201989\%20Nominal\%20Reference.pdf.

Krifka, Manfred. 1992. Thematic relations as links between nominal reference and temporal constitution. In Ivan A. Sag \& Anna Szabolcsi (eds.), Lexical matters, 29-53. Stanford, CA: CSLI Publications. http://amor.cms.hu-berlin.de/ h2816i3x/Publications/Krifka\%201992\%20Thematic\%20Relations.PDF.

Krifka, Manfred. 1998. The origins of telicity. In Susan Rothstein (ed.), Events and grammar, vol. 70 Studies in linguistics and philosophy, 197-235. Dordrecht, Netherlands: Kluwer. doi:10.1007/978-94-011-3969-4_9.

Link, Godehard. 1983. The logical analysis of plurals and mass terms: A latticetheoretical approach. In Reiner Bäuerle, Christoph Schwarze \& Arnim von Stechow (eds.), Meaning, use and interpretation of language, 303-323. Berlin, Germany: de Gruyter. doi:10.1515/9783110852820.302. Reprinted in Link (1998), Chapter 1.

Link, Godehard. 1998. Algebraic semantics in language and philosophy. Stanford, CA: CSLI Publications.

Mittwoch, Anita. 1982. On the difference between eating and eating something: activities versus accomplishments. Linguistic Inquiry 13(1). 113-122. http: //www.jstor.org/stable/4178263.

Moltmann, Friederike. 1991. Measure adverbials. Linguistics and Philosophy 14(6). 629-660. doi:10.1007/bf00631962.

Naumann, Ralf. 1999. A dynamic logic of events and states for the interaction between plural quantification and verb aspect in natural language. Logic Journal of the IGPL 7(5). 591-627. doi:10.1093/jigpal/7.5.591.

Piñón, Christopher. 2015. An illusory common core. Theoretical Linguistics 41(3-4). 185-195. doi:10.1515/tl-2015-0012.

Väänänen, Jouko. 2007. Dependence logic: A new approach to independence friendly logic, vol. 70 London Mathematical Society Student Texts. Cambridge, UK: Cambridge University Press. doi:10.1017/cbo9780511611193.

Verkuyl, Henk J. 1972. On the compositional nature of the aspects. Dordrecht, Netherlands: Reidel. doi:10.1007/978-94-017-2478-4.

White, Michael. 1994. A computational approach to aspectual composition. 
Philadelphia, PA: University of Pennsylvania dissertation.

Winter, Yoad. 2001. Flexibility principles in Boolean semantics: The interpretation of coordination, plurality, and scope in natural language. Cambridge, MA: MIT Press.

Zucchi, Sandro \& Michael White. 2001. Twigs, sequences and the temporal constitution of predicates. Linguistics and Philosophy 24(2). 223-270. doi:10.1023/a:1005690022190.

Zweig, Eytan. 2009. Number-neutral bare plurals and the multiplicity implicature. Linguistics and Philosophy 32(4). 353-407. doi:10.1007/s10988-009-9064-3.

Lucas Champollion

Department of Linguistics

New York University

10 Washington Place

New York, NY 10003

champollion@nyu.edu

Haoze Li

Department of Linguistics

New York University

10 Washington Place

New York, NY 10003

haozeli@nyu.edu
Justin Bledin

Department of Philosophy Johns Hopkins University

206 Gilman Hall

3400 N. Charles Street

Baltimore, MD 21218

jbledin@jhu.edu 